\title{
Telescope Array Experiment
}

\author{
Shoichi Ogio $^{1, *}$ for the Telescope Array collaboration \\ ${ }^{1}$ Graduate School of Science, Osaka City University, Osaka, Osaka, Japan
}

\begin{abstract}
The Telescope Array is the largest hybrid cosmic ray detector in the Northern hemisphere designed to measure primary particles in $4 \mathrm{PeV}$ to $100 \mathrm{EeV}$ range. The main TA detector consists of an air shower array of 507 plastic scintillation counters on a $1.2 \mathrm{~km}$ square grid and fluorescence detectors at three stations overlooking the sky above the air shower array. The experiment and its recent measurements - spectrum, composition, and anisotropy - is reviewed. Recently the construction of the TA Low energy Extension (TALE) detector, which consists of an additional fluorescence detector and an infill array, was finished. TALE lowers the energy threshold of TA down to $4 \mathrm{PeV}$. We are also constructing the TAx4 detector to increase statistics in particular at the highest energies. The current status and the future prospects of these new TAx 4 experiments is reported.
\end{abstract}

\section{Introduction}

Telescope Array (TA) is an observatory for ultra high energy cosmic rays in the northern hemisphere. Operating TA together with the TA Low energy Extension (TALE) detectors, we archive a vary wide target energy range from $10^{16}$ to $10^{20} \mathrm{eV}$. The next section is a briefly introduction of the Telescope Array experiment, such as our detectors and analysis. In section 3 , I will report recent results on the energy spectrum, chemical composition and anisotropy studies. Section 4 is an introduction and status report of on-going extension projects called TA $\times 4$ and TALE.

\section{The Telescope Array Detectors}

The TA site is located in the desert about $1400 \mathrm{~m}$ above sea level centered at $39.3^{\circ} \mathrm{N}$ and $112.9^{\circ} \mathrm{W}$ in Millard county, Utah, USA, about $200 \mathrm{~km}$ southwest of Salt Lake City. A control center to support operations of the TA, TALE and $\mathrm{TA} \times 4$ instruments is in the city of Delta located near the northeast side of the TA SD array.

Figure 1 is a map of the TA site, and the dots and markers indicate the locations of detectors. Figure 2 shows some of the infrastructure. The TA surface detector (SD) array consists of 507 scintillation detectors of 3 square meter size deployed with $1.2 \mathrm{~km}$ spacing. The coverage area by the SD array is about $700 \mathrm{~km}^{2}$. The sky above the array is observed by 38 fluorescence detectors (FDs), installed in three stations which are called Middle Drum (MD), Black Rock Mesa (BRM) and Long Ridge (LR) stations. The arrows in Figure 1 indicate the border of the field of view (FOV) of each FD station. The telescopes in the MD station were arranged to view $120^{\circ}$ in azimuth and $3^{\circ}-33^{\circ}$ in elevation. Comparing with the MD station, the azimuthal

\footnotetext{
*e-mail: sogio@sci.osaka-cu.ac.jp
}

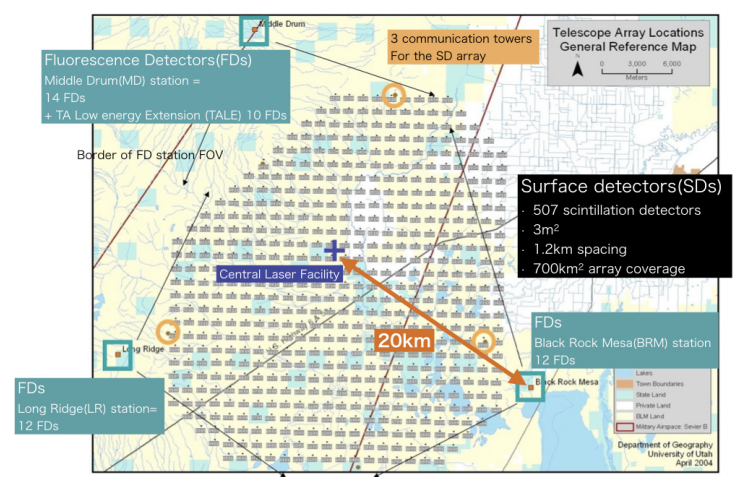

Figure 1. The map of the TA site. The labeled black dots indicate the location of SDs, and the green open square is the positions of the FD stations. The three open orange markers indicate the location of the communication towers.

FOV of the BRM and the LR stations is narrow and $108^{\circ}$. At the center of the array, there is the central laser facility (CLF) used for the calibrations of FDs. The distance between the CLF and each FD station is about $20 \mathrm{~km}$.

The key feature of our experiment is stereo-hybrid observations of air showers. It means the simultaneous observation of an air shower with multiple FD stations and also with the SD array. The stereoscopic measurement with multiple FD stations or the hybrid measurement with an FD stations and an SD improve the determination accuracy of the shower geometry. As a result, the determination accuracy on the primary energy and on the depth of maximum development of showers (Xmax) are improved. 


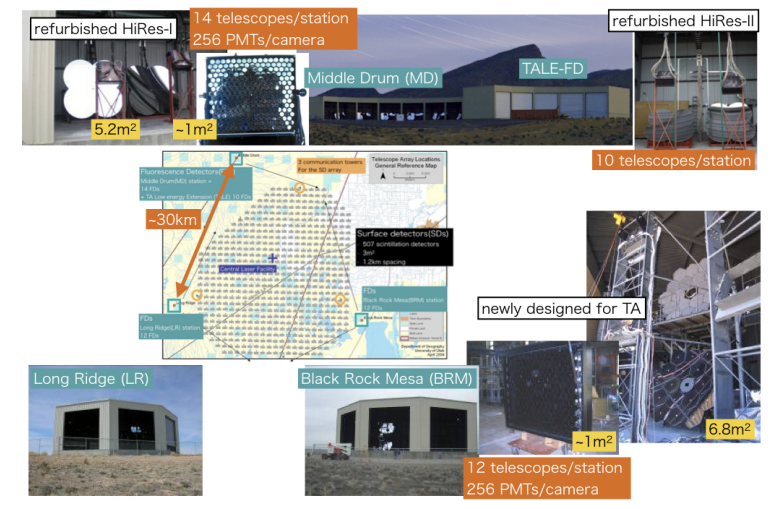

Figure 2. Pictures of the four FD stations and of the telescopes and cameras in the stations.

\subsection{Fluorescence Detectors}

The MD station has 14 telescopes, each of which consists of a refurbished telescope used in HiRes-I [1], a camera comprising 256 PMTs. Each telescope unit uses sampleand-hold electronics with a $5.6 \mu$ s gate.

Just beside the MD station, there is the TALE-FD station which has ten refurbished HiRes-II [1] telescopes observing higher elevation angles which means measuring lower energies, than TA. These are the refurbished HiResII telescopes. A camera of 256 pixels is placed at the focal plane of the mirror. Each pixel covers a one degree cone in the sky, and each camera has a FOV of $16^{\circ}$ in azimuth by $14^{\circ}$ in elevation. The PMT signals are recorded by a 10 $\mathrm{MHz}$ FADC readout system with an 8-bit resolution. Analog sums over rows and columns of pixels, also sampled at 8-bits, allow recovery of saturated PMTs in most cases.

The systems in the LR and the BRM stations are exactly the same, and it was newly designed for the TA experiment [2]. Each station has 12 telescopes, and each telescope has 256 PMTs focused onto a $6.8 \mathrm{~m}^{2}$ mirror for light collection. Each PMT's analog signals are digitized by FADC electronics which employ 12 bit digitizers operating at $40 \mathrm{MHz}$. Before storage to the DAQ, four digital samples are summed to provide an equivalent 14 bit, 10 $\mathrm{MHz}$ sampling rate providing a time resolution of $100 \mathrm{~ns}$.

The process of analysis consists of four steps: PMT selection, shower geometry reconstruction, reconstruction of longitudinal shower profile and quality cuts. An FD monocular plane fitting procedure determines the geometry of the air shower track relative to the observing FD. At first the shower-detector plane (SDP) normal vector is found from the number of tubes along the shower track, the pointing directions and the tube signals of each tube in the SDP. In the monocular reconstruction, once the SDP is known, the shower impact parameter and the SDP angle are calculated by comparing the observed tube trigger times and the expected trigger times. Using the information from the two stations simultaneously allows the "stereo reconstruction". For the stereo analysis the shower geometry is determined primarily by finding the intersection of the two SDPs. The other idea called "hybrid re-

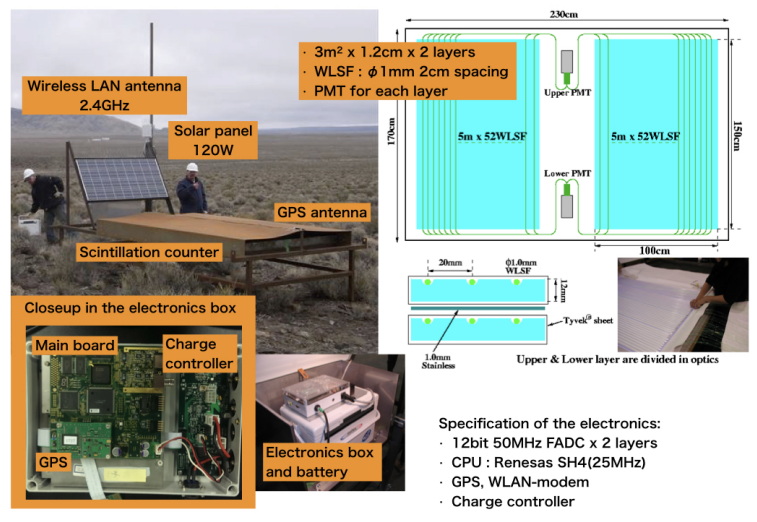

Figure 3. Pictures of a surface detector and electronics.

construction" is the use of timing information from one or more SDs in addition to the FD tube timings. The SD timing at which the shower plane crosses the ground gives an "anchor" in the conventional FD timing fit. The stereo or hybrid reconstructions significantly improve the accuracy in shower geometry determination compared to that of the FD monocular mode.

Once the shower geometry is determined, the shower profile including the depth of shower maximum (Xmax) determined using the light profile observed by the FDs by the inverse Monte Carlo method, and then finally the primary energy is found by integrating the shower profile.

\subsection{Surface Detector Array}

The SD array consists of 507 detector units, which were deployed in a square grid with $1.2 \mathrm{~km}$ spacing to cover a total area of approximately $700 \mathrm{~km}^{2}$. Each surface detector has a scintillation counter, a solar panel, a wireless LAN and GPS antennas, and controlling electronics and a battery behind the solar panel on a steel platform (see Fig. 3). The scintillation counter consists of two layers of plastic scintillator. Each layer of scintillator has an area of $3 \mathrm{~m}^{2}$ and a thickness of $1.2 \mathrm{~cm}$. Scintillation light is collected through 104 wavelength-shifting (WLS) fibers, and both ends of the fibers from a layer are bundled together and connected to a PMT. The controlling electronics consists of 12 bit $50 \mathrm{MHz}$ FADCs, a CPU, a GPS module, a wireless LAN modem and a charge controller.

The process of analysis for SD array data consists of three steps (see Fig. 4). At first a time fit of shower arrivals at SDs is performed to determine the geometry of the cosmic ray air shower. The lateral distribution of charged particle densities at the SDs is fit using the AGASA lateral distribution function [3, 4] to determine S800, the density of shower particles at a lateral distance of $800 \mathrm{~m}$ from the air shower axis. The primary energy of the cosmic ray is estimated by using a look-up table in S800 and the shower zenith angle. The table is obtained by a large statistics MC simulation using CORSIKA and the QGSJET II-03 hadronic model. Finally, the reconstructed energy by the $\mathrm{SD}$ analysis is scaled to the energy measured by the TA 


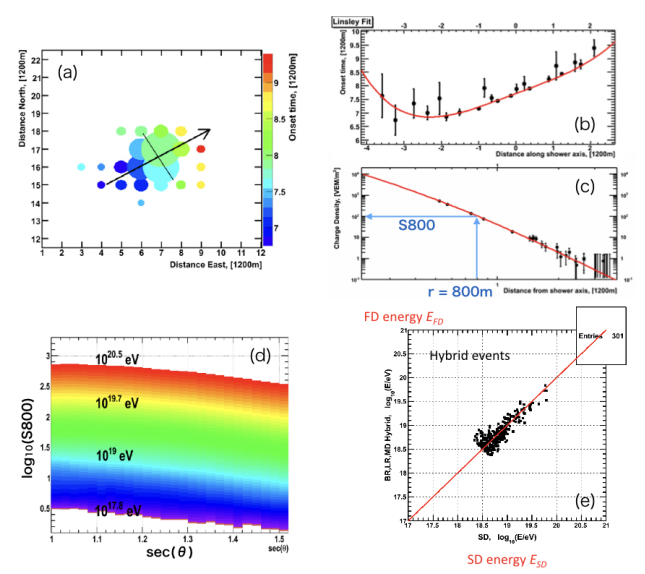

Figure 4. The process of analysis for SD array data

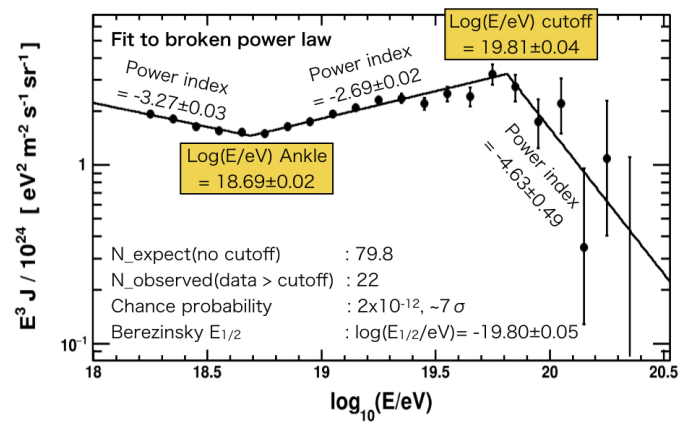

Figure 5. The nine year spectrum measured by the TA SD array [6]

FDs, which is determined using calorimetric detection of an air shower energy deposition in the atmosphere with less hadronic interaction dependence than the SD [5].

\section{Recent Results}

\subsection{Energy spectrum and related studies}

Figure 5 is the nine year spectrum measured by the TA SD array, and the data points fit to a broken power law [6]. There are two breaks, the ankle at $10^{18.69} \mathrm{eV}$ and a cutoff at $10^{19.81} \mathrm{eV}$. The significance of the existence of the cutoff is about seven sigma.

The Auger-TA spectrum working group showed a comparison between Auger and TA SD spectra, which do not overlap each other by about $10 \%$ energy shift. However, this shift is well within the stated systematic energy scale uncertainties by Auger 14\% and TA 21\%. When the Auger energies are raised by $5.2 \%$ and TA energies lowered by $5.2 \%$, as shown in Figure 6(a), the fluxes by two experiment well overlap below $10^{19.4} \mathrm{eV}$. Nevertheless, the two fluxes do not overlap each other at energies higher than $10^{19.4} \mathrm{eV}$. However, if we use data only from the common declination band, that is from -15.9 degree to
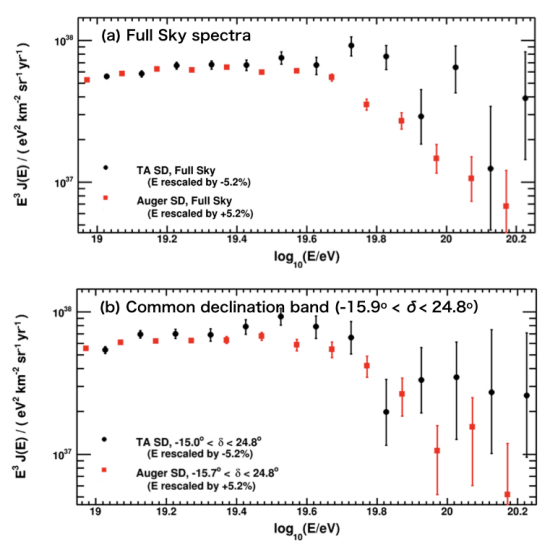

Figure 6. A comparison between TA SD full sky spectrum with energies scaled by $-5.2 \%$ and Auger SD full sky spectrum with energies scaled by $+5.2 \%$ [7]. (a) is a comparison of the full sky spectra, and (b) is a comparison of the spectra for the common declination band.

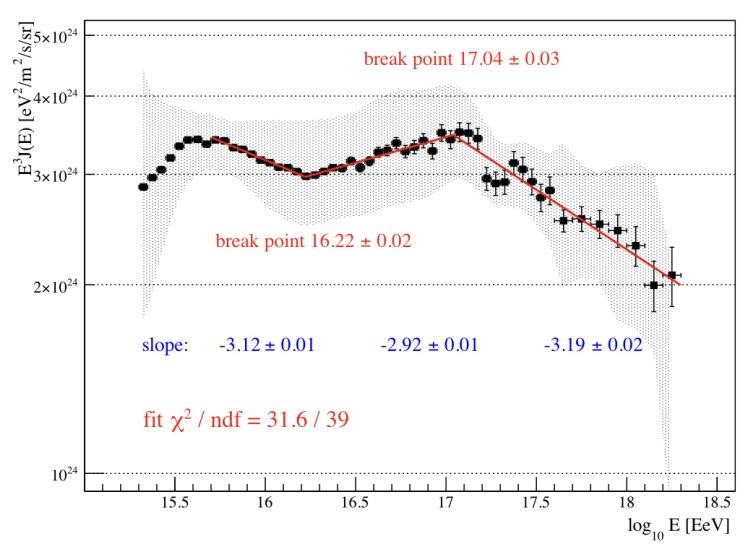

Figure 7. TALE cosmic rays energy spectrum measured with 22 months of data [8].

24.8 degree, we can see a better agreement between Auger and TA as shown in Figure 6(b).

Figure 7 is the two year spectrum in the lower energy range, from $10^{15.3} \mathrm{eV}$ to $10^{18} \mathrm{eV}$, measured by the TALE FDs, and the data points fit to a broken power law [8]. There are two breaks, a break at $10^{16.22}$ and the second knee at $10^{17.04} \mathrm{eV}$. We note that since the estimation of exposure strongly depends on the chemical composition in particular in the lower energy region, the energy spectrum also depends on an assumed chemical composition. For Figure 7 a mixed primary composition representing the reconstructed Xmax distribution is assumed. The gray band indicates the size of the systematic uncertainties.

\subsection{Chemical Composition}

FIgure 8 is the mean $\mathrm{Xmax},<\mathrm{Xmax}>$, as a function of energy as observed by TA in BRM and LR hybrid mode over 8.5 years of data collection. The numbers above the data points indicate the number of events observed. The gray band is the systematic uncertainty of this 


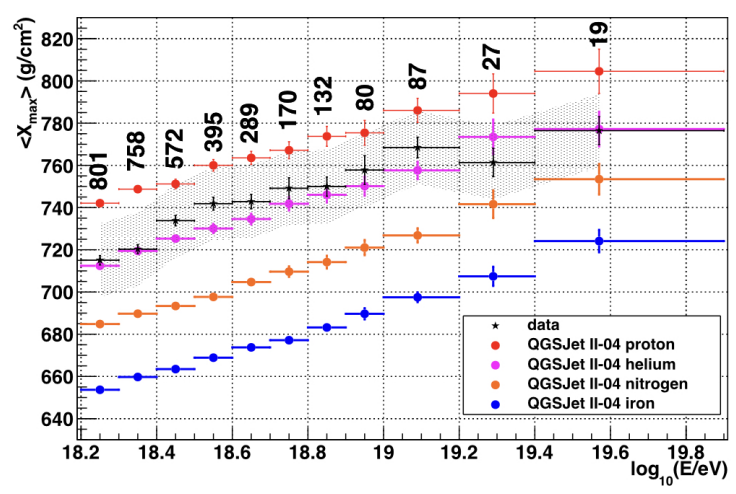

Figure 8. Mean Xmax as a function of energy as observed by TA in BRM and LR hybrid mode over 8.5 years of data collection [9].

analysis. Reconstructed Monte Carlo of four different primary species generated using the QGSJet II-04 hadronic model are shown for comparison [9]. Within systematic uncertainties, $<\mathrm{Xmax}>$ of the data is in agreement with QGSJet II-04 protons and helium for nearly all energy bins. There is a clear separation between the region of systematic uncertainty and heavier elements such as nitrogen and iron.

The reconstructed Xmax distribution for a single element, such as QGSJet II-04 protons is sampled according to the same number of events recorded in the data for a given energy bin. $<\mathrm{Xmax}>$ and the standard deviation of Xmax distribution, $\sigma(\mathrm{Xmax})$, of the energy are calculated and recorded for this sample. This procedure is then repeated 5000 times. The distribution of $<\mathrm{Xmax}>$ and $\sigma(\mathrm{Xmax})$ is used to calculate the $68.3 \%, 90 \%$, and 95\% confidence intervals. The entire $<\mathrm{Xmax}>$ and $\sigma(\mathrm{Xmax})$ calculated by this method is then plotted as a 2-dimensional distribution along with the computed confidence intervals. This procedure is repeated for the other three chemical elements used in the analysis. Figures 9 shows this measurement for all observed energy bins. The $<\mathrm{Xmax}>$ and $\sigma(\mathrm{Xmax})$ of the data observed in each energy bin is also recorded as a single red star. Additionally, the statistical, systematic, and combined statistical and systematic error bounds are marked around the data. Figures 9(a) $-9(\mathrm{f})$, corresponding to the energy range $10^{18.2}-10^{18.8} \mathrm{eV}$ show $\sigma(\mathrm{Xmax})$ of the data to resemble QGSJet II-04 protons, and $<$ Xmax $>$ of the data falls within the $68.3 \%$ confidence interval of the proton distributions within the data's systematic uncertainty.

Figure $9(\mathrm{~g})$, corresponding to the energy range $10^{18.8}$ $10^{18.9} \mathrm{eV}$, shows that the $68.3 \%$ confidence intervals of QGSJet II-04 proton and helium both fall within the bounds of the systematic uncertainty of the data. In Figure 9 (h), corresponding to the energy range $10^{18.9}-10^{19.0} \mathrm{eV}$, $\sigma(\mathrm{Xmax})$ of the data fluctuates up from the previous energy bin and the systematic error bounds of the data falls within the $68.3 \%$ confidence interval of protons. In Figure 9(i), the $68.3 \%$ confidence intervals of both proton and he-

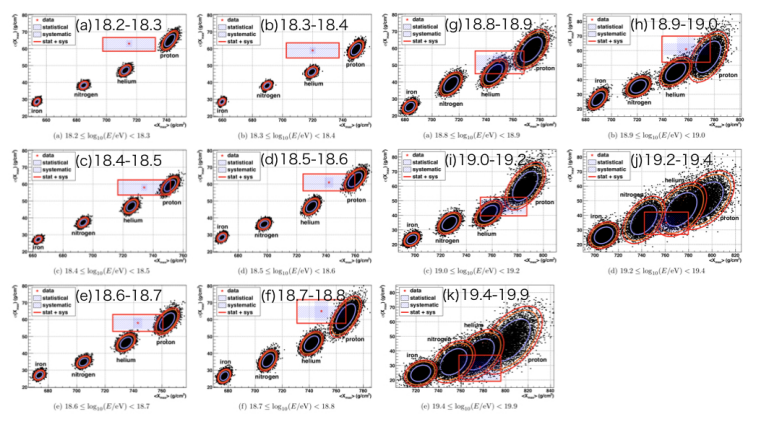

Figure 9. Measurements of data and QGSJet II-04 Monte Carlo $<$ Xmax $>$ and $\sigma$ (Xmax). Each Monte Carlo chemical element shows the $68.3 \%$ (blue ellipse), $90 \%$ (orange ellipse), and 95\% (red ellipse) confidence intervals.

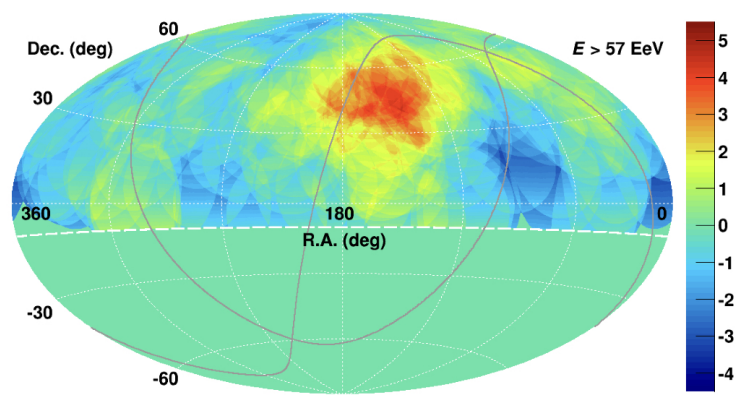

Figure 10. Significance map from $9 \mathrm{yr}$ TA-SD events $\mathrm{E}>57 \mathrm{EeV}$ with $25^{\circ}$ oversampling radius.

lium fall within the bounds of the systematic uncertainty of the data. Figures Figure 9(j) and 9(k) show that within the data's systematic uncertainty the data may resemble QGSJet II-04 proton, helium, or nitrogen.

\subsection{Arrival Direction Distributions}

In the highest energy set with $\mathrm{E}>57 \mathrm{EeV}$ collected during the first 5 years of the TA operation, a concentration of events has been observed in the circle of radius $20^{\circ}$ around the direction $\mathrm{RA}=146.7^{\circ}, \mathrm{DEC}=43.2^{\circ}$ [10]. The number of events has been almost doubled now, and we scanned the oversampling radius from $15^{\circ}$ to $35^{\circ}$ with $5^{\circ}$ interval. We found the maximum significant excess appears at $\mathrm{RA}=144.3^{\circ}, \mathrm{DEC}=40.3^{\circ}$ with the $25^{\circ}$ oversampling radius as shown in Figure 10. The statistical significance is $5.1 \sigma$, and the probability of such a hotspot appearing by chance in an isotropic cosmic-ray sky is $3.0 \sigma$. 


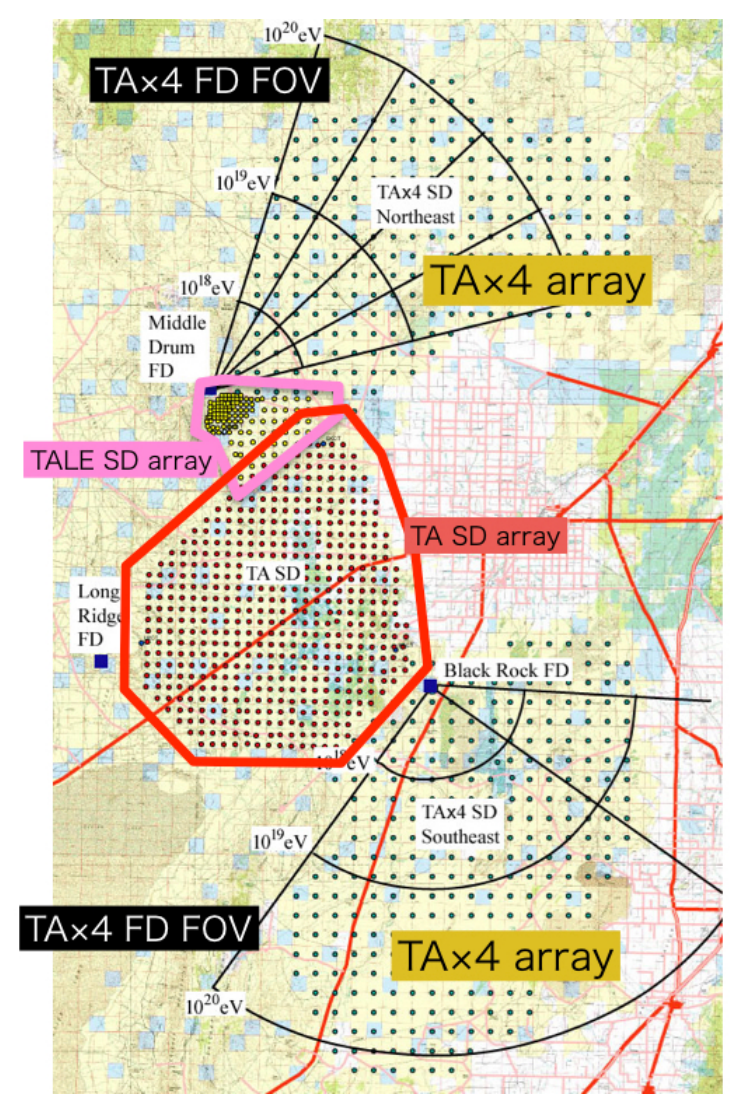

Figure 11. This map shows the overview of the TA site. Each green circle in the northeast and southeast corresponds to the planned location of each TAx4 SD. The spacing of TAx4 SD is $2.08 \mathrm{~km}$. The red circle in the west shows the location of TA SD. The spacing of TA SD is $1.2 \mathrm{~km}$. The 2 fan shapes drawn with black lines describe the expected field of view from TAx4 FDs. Four telescopes of FD will be built in the north Middle Drum site and 8 telescopes of FD will be built in the south Black Rock site. The overlap of the locations of SD and the field view of FD enables SD and FD hybrid observation.

\section{Extension of Telescope Array}

\subsection{TA $\times 4$}

New SDs and FDs are planned to be constructed for the TAx 4 experiment to cover 4 times larger area than TA, about $3000 \mathrm{~km}^{2}$, to observe cosmic rays especially with the highest energies using high statistics (Fig. 11). This project is expected to clarify not only the source of the hotspot but also the energy spectrum and the composition at the highest energies. The five-year proposal for $\mathrm{TA} \times 4$ SD was accepted in the spring of 2015. The proposal for constructing 2 FD stations was also accepted in 2016. In our plan, 500 SDs are going to be made and deployed with $2.08 \mathrm{~km}$ spacing. After 3 years construction work, 180 SDs in Utah and the next 60 are to be prepared at ICRR and SKKU in 2018. For two FD stations with refurbished twelve HiRes telescopes, telescopes and electronics were prepared at the University of Utah, and the northern stations had been installed and observed the first light in 2018. The site construction is underway at the southern site.

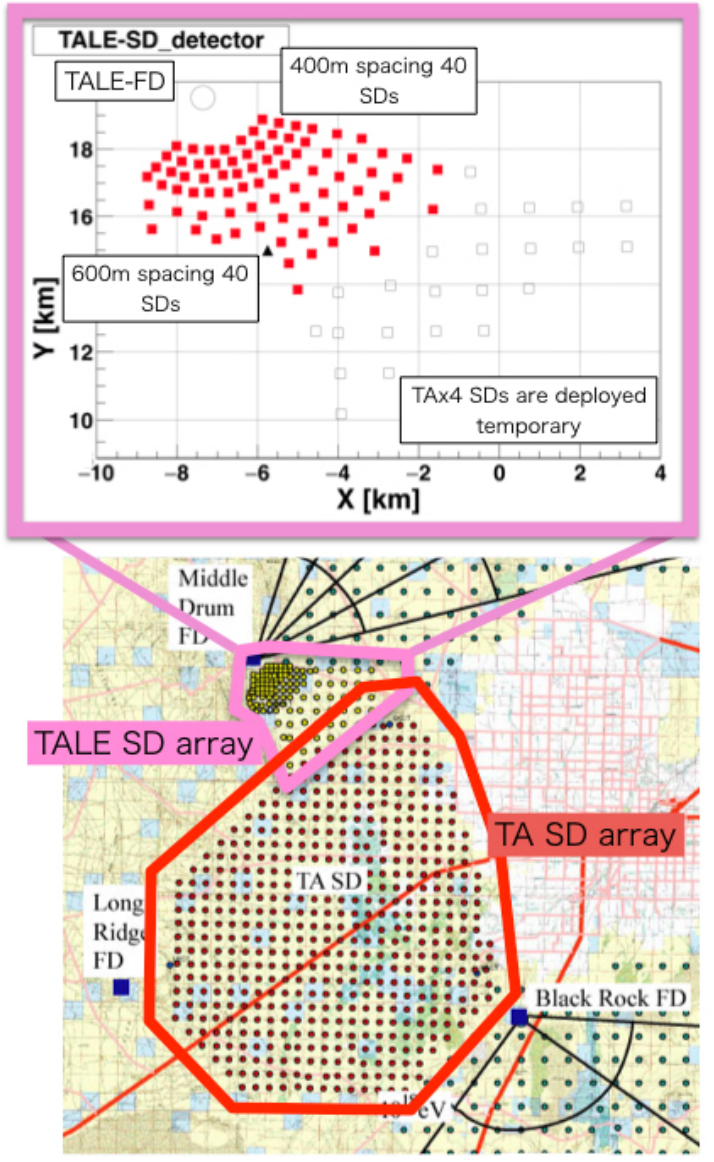

Figure 12. A map of the TA experiment site and a close up of TALE site are shown.

\subsection{TA Low energy Extension (TALE)}

The Telescope Array Low-energy Extension (TALE) experiment is a hybrid air shower detector for observation of air showers produced by very high energy cosmic rays above $10^{16.5} \mathrm{eV}$. TALE is located at the TA site (see Fig. 12). TALE has a SD array made up of 80 scintillation counters (40 with $400 \mathrm{~m}$ spacing, 40 with $600 \mathrm{~m}$ spacing) and a FD station consisting of ten FD telescopes located at the TA Middle Drum station. TALE-FD full operation started in 2013. The deployment of 80 SDs and the installations of DAQ/control electronics had been completed by February 2018.

\section{Acknowledgements}

The Telescope Array experiment is supported by the Japan Society for the Promotion of Science (JSPS) through Grants-in-Aid for Priority Area 431, for Specially Promoted Research JP21000002, for Scientific Research (S) JP19104006, for Specially Promoted Research JP15H05693, for Scientific Research (S) JP15H05741 and for Young Scientists (A) JPH26707011; by the joint research program of the Institute for Cosmic Ray Research (ICRR), The University of Tokyo; by the U.S. National Science Foundation 
awards PHY-0601915, PHY-1404495, PHY-1404502, and PHY-1607727; by the National Research Foundation of Korea (2016R1A2B4014967, 2016R1A5A1013277, 2017K1A4A3015188, 2017R1A2A1A05071429); by the Russian Academy of Sciences, RFBR grant 16-02-00962a (INR), IISN project No. 4.4502.13, and Belgian Science Policy under IUAP VII/37 (ULB). The foundations of Dr. Ezekiel R. and Edna Wattis Dumke, Willard L. Eccles, and George S. and Dolores Doré Eccles all helped with generous donations. The State of Utah supported the project through its Economic Development Board, and the University of Utah through the Office of the Vice President for Research. The experimental site became available through the cooperation of the Utah School and Institutional Trust Lands Administration (SITLA), U.S. Bureau of Land Management (BLM), and the U.S. Air Force. We appreciate the assistance of the State of Utah and Fillmore offices of the BLM in crafting the Plan of Development for the site. Patrick Shea assisted the collaboration with valuable advice on a variety of topics. The people and the officials of Millard County, Utah have been a source of steadfast and warm support for our work which we greatly appreciate. We are indebted to the Millard County Road Department for their efforts to maintain and clear the roads which get us to our sites. We gratefully acknowledge the contribution from the technical staffs of our home institutions. An allocation of computer time from the Center for High Performance Computing at the University of Utah is gratefully acknowledged.

\section{References}

[1] R. U. Abbasi et al., PRL 92, 151101(2004)

[2] H. Tokuno et al., NIM-A 601, 364(2009)

[3] M. Takeda et al., PRL 81, 1163(1998)

[4] M. Takeda, Astropart. Phys. 19, 447(2003)

[5] T. Abu-Zayyad et al., ApJL 768, L1(2013)

[6] J. N. Matthews, ICRC2017, CRI172(2017)

[7] D. Ivanov, ICRC2017, CRI231(2017)

[8] R. U. Abbasi et al., ApJ, in press; arXiv: 1803.01288(2018)

[9] R. U. Abbasi et al., ApJ 858, 76(2018)

[10] R. U. Abbasi et al., ApJL 790, L21(2014) 